\title{
The Single-trial Analysis of P300 and the Difference Threshold of Modulated Vibration
}

\author{
Reiko Ohira Non-member (The Unibersity of Electro-Communications) \\ Masahumi Uchida Member (The Unibersity of Electro-Communications, uchi@ee.uec.ac.jp) \\ Akinori Nozawa Member (Aoyama Gakuin Unibersity, akio@ee.aoyama.ac.jp) \\ Hideto Ide Member (Aoyama Gakuin Unibersity, ide@ee.aoyama.ac.jp)
}

Keywords: tactile sensor, modulated vibration, difference-threshold, P300, single-trial analysis

This study focuses on the tactile display that uses the modulated vibration. Modulated vibration includes two types of single-frequency vibration: one with a short cycle $T_{s}$ and one with a long cycle $T_{l}$. The modulated vibration is represented as the dots in the $T_{s}^{-1}$ vs. $T_{l}^{-1}$ plane, and information can be obtained by changing $T_{s}$ or $T_{l}$.

The first challenge is to specify the different-threshold (DT) about frequency for a human's tactile sensor to the modulated vibration when creating the display. Psychological or ERP (eventrelated potential) measurements have been used to estimate the DT on the $T_{s}^{-1}$ vs. $T_{l}^{-1}$ plane using a task in which the subject must recognize the difference between an NT (non-target) and a TA(target), where the TA is a more rare stimulus than the NT.

This paper proposes a single-trial analysis for ERP measurements. ERP appears in EEGs (electroencephalogram) when human are performing work, such as recognizing or remembering external stimuli. This study focuses on the ERP's P300 wave that appears when a human cognitively processes an external stimulus. P300 is typically detected using averaged EEG analysis, but the disadvantage of this method is that the EEG wave must be averaged over hours and over time a human concentration tends to deteriorate. However, previous attempts to detect P300 with a singletrial analysis have proved inconclusive. T-test theory was used in the proposed analysis based on the assumption that, on average, the P300' $\mathrm{s}$ voltage should be higher than that of background activity in a single-trial EEG because P300 has been detected at the top of the plus-voltage in averaged EEGs.

The experiment was conducted with five subjects, and the DTs were individually determined. The subjects were given the task of distinguishing stimuli varied between NT and TA, were $\mathrm{NT}\left(T_{s}^{-1}, T_{l}^{-1}\right)=(120,8)[\mathrm{Hz}]$ and an optional TA was included.

As a result, the belt-shape figure evident in all five subject model data. In this report, $t_{A}$ means before-time of begining of the stimulus, and $t_{B}$ means after-time of that. The shape

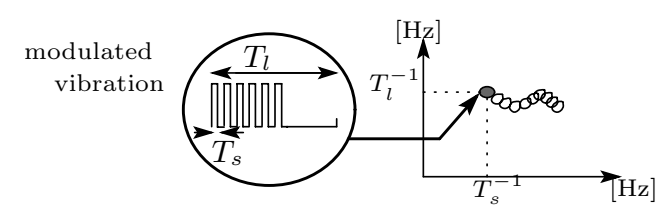

Fig. 1. The $T_{s}^{-1}$ vs. $T_{l}^{-1}$ plane is especially remarkable in $300 \leq t_{A} \leq 500[\mathrm{~ms}]$. In short, the effectiveness of manipulating T-test data to detect P300 in single-trial EEGs was assured. In addition, it was discovered that the DT of the proposed method correlates with those reported by previous methods by calculating the correlation coefficients.

According to results, the effectiveness of presuming DT using a single-trial analysis of P300 was assured. Future studies will focus on detecting DT for various tasks using the method proposed in this report, and the practical use of the tactiledisplay that employs modulated vibration will be developed. Lastly, the authors would like to thank all of the people for their cooperation.

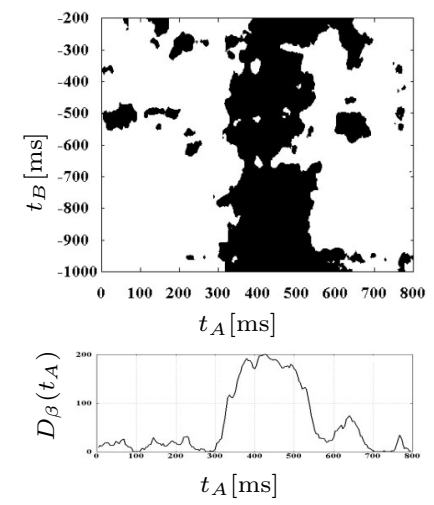

(1) When TA was given
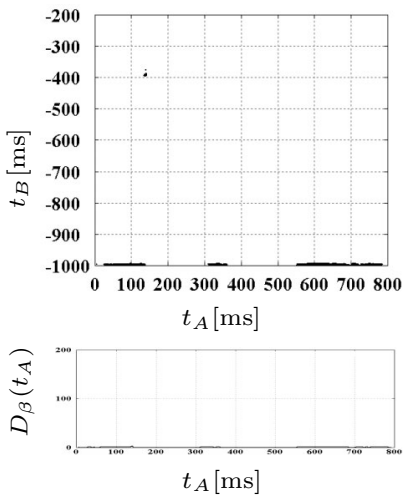

(2) When NT was given
Fig. 2. The Model data of subject (a)

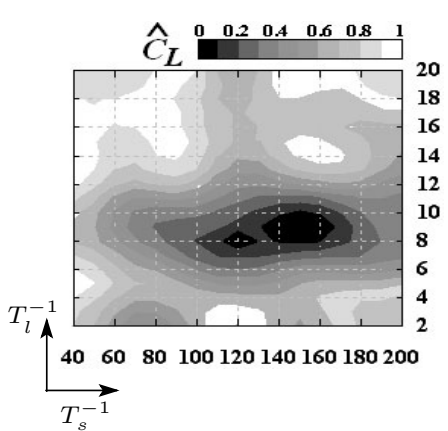

(a) Single-trial analysis

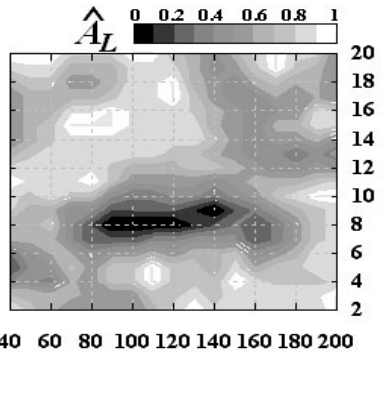

(b) The psychophysical measurement
Fig. 3. The subject (a)'s DT 


\section{The Single-trial Analysis of P300 and the Difference Threshold of Modulated Vibration}

$\begin{array}{ll}\text { Reiko Ohira* } & \text { Non-member } \\ \text { Masahumi Uchida* }^{*} & \text { Member } \\ \text { Akio Nozawa** } & \text { Member } \\ \text { Hideto Ide }^{* *} & \text { Member }\end{array}$

This study reports the estimation of different-threshold (DT) when humans recognize various modulated vibrations using their tactile sensor. The modulated vibration consists of two frequency components. A psychological or the event related potential (ERP) measurement was used to estimate DT. ERP appears in an electro-encephalogram (EEG) when a human recognizes the information emitted by an external stimulus, and this study detects P300, which is a component of ERP.

This paper proposes a single-trial analysis that uses the T-test, which deviates from the conventional method for detecting P300 called the averaged EEG method. Finally, the proposed method estimates DT. As a result, the effectiveness of the proposed method is assured by estimating DTs on five subjects.

Keywords: tactile sensor, modulated vibration, difference-threshold, P300, single-trial analysis

\section{Introduction}

This study focuses on the tactile display that uses the modulated vibration ${ }^{(1)}$. Modulated vibration includes two types of single-frequency vibration: one with a short cycle $T_{s}$ and one with a long cycle $T_{l}$. A graphical depiction of the $T_{s}^{-1}$ vs. $T_{l}^{-1}$ plane is provided in Fig.1, and the modulated vibration is represented as the dots in the plane. Two-dimensional information can be obtained by changing $T_{s}$ or $T_{l}$.

The first challenge is to specify the different-threshold (DT) about frequency for a human's tactile sensor to the modulated vibration when creating the display. Psychological or ERP (eventrelated potential) measurements have been used to estimate the DT on the $T_{s}^{-1} v s . T_{l}^{-1}$ plane using a task in which the subject must recognize the difference between an NT (non-target) and a TA (target) ${ }^{(2)}{ }^{(3)}$, where the TA is a more rare stimulus than the NT.

This paper proposes a single-trial analysis for ERP measurements. ERP appears in EEGs (electroencephalogram) when a human is performing work, such as recognizing or remembering external stimuli. This study focuses on the ERP's P300 wave that appears when a human cognitively processes an external stimulus. P300 is typically detected using averaged EEG analysis, but the disadvantage of this method is that the EEG wave must be averaged over hours ${ }^{(4)-(6)}$ and over

\footnotetext{
* Department of Electoronic Engineering, The University of Electro-Communications

1-5-1, Chohugaoka, Chouhu 182-8585

** Department of Electrical Engineering and Electronics, Aoyama Gakuin University

5-10-1, Fuchinobe, Sagamihara 229-8558
}

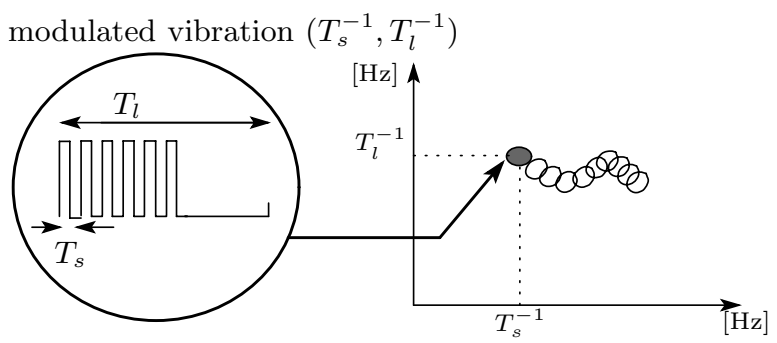

Fig. 1. The $T_{s}^{-1}$ vs. $T_{l}^{-1}$ plane

time a human's concentration tends to deteriorate. However, previous attempts to detect P300 with a single-trial analysis have proved inconclusive ${ }^{(7)-(9)}$.

This paper proposes a new single-trial analysis that employs T-test theory and estimates the DT. The remainder of the paper is organized as follows. The singletrial analysis technique and the method for determining DT is described in Section 2. Section 3 shows the experimental setup and the EEG measurement, and the results and discussion are presented in Section 4. Finally, Section 5 reiterates the conclusions and discusses the future course of study.

\section{The Method to Estimate DT}

The P300 level used to estimate DT is determined using a single-trial analysis called "cognition-level" in this report.

2.1 The Single-trial Analysis Generally speaking, P300 is detected from the time when the top plusvoltage is specified in an averaged EEG. Thus, it is assumed that, on average, the P300 voltage for a singletrial EEG is higher than that for an EEG that does not 


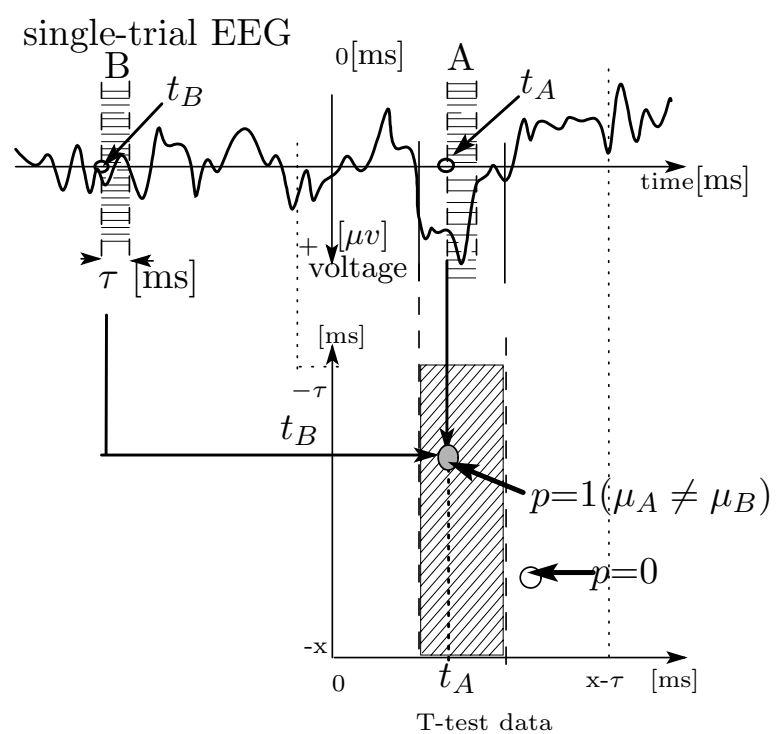

Fig. 2. Outline of the single trial analysis

have P300 ${ }^{(10)}$. This study uses the T-test method for the single-trial analysis, which is then used to estimate $\mathrm{DT}^{(11)}$.

Figure 2 shows the outline for the single-trial analysis. A single-trial EEG recorded for $2 x[\mathrm{~ms}]$, from $-x[\mathrm{~ms}]$ to $+x[\mathrm{~ms}]$, was set to $0[\mathrm{~ms}]$ at the start-time for the stimulus. First, two groups (A and B) were obtained from $t_{A}$ or $t_{B}$ in the EEG, and the data was recorded for $\tau[\mathrm{ms}] . \quad 0 \leq t_{A} \leq x-\tau[\mathrm{ms}]$ and $-x \leq t_{B} \leq \tau[\mathrm{ms}]$ ranges were fixed $\mu_{A}$ and $\mu_{B}$ were assumed to be the averages of groups $\mathrm{A}$ and $\mathrm{B}$, respectively. Second, a Ttest is performed on the group and the significance-level for this report was determined to be $\alpha[\%]$. If $\mu_{A} \neq \mu_{B}$, $p=1$ is assumed, otherwise $p=0$. This relationship is depicted in Eq.(1). $p$ can be expressed in the $t_{A} v s . t_{B}$ plane, referred to as the "T-test data" plane. In this paper, a black-dot on is plotted on the plane when $p=1$, otherwise a white dot is plotted. If P300 appeared in the single-trial EEG, the T-test data would assume the characteristic belt-shape pattern on the $t_{B}$ axis.

$$
p\left(T_{s}^{-1}, T_{l}^{-1}, t_{A}, t_{B}\right)=\left\{\begin{array}{ll}
1, & \text { if } \mu_{A} \neq \mu_{B} \\
0, & \text { otherwise }
\end{array} \ldots\right.
$$

2.2 Estimation of DT Using the experimental data, the model-data that contains the P300's feature in the $t_{A}$ vs. $t_{B}$ plane was determined, and this model-data is used to estimate the cognition-level.

Figure 3 shows the method employed to create the model-data. When there are N EEGs that contain P300, stimulus $\left(T_{s}^{-1} k, T_{l}^{-1} k\right), k=1,2, \cdots, N$ is introduced and T-test data are obtained as $p_{k}\left(t_{A}, t_{B}\right)$. Then, the modeldata are obtained as $P_{\beta}$ function by using Eq.(2).

The experimental $p$ values are compared with the $P_{\beta}$ using the $C_{L}$ function in Eq.(3). In this paper, $\widehat{C}_{L}$ is employed and smoothed on the $T_{s}^{-1} v s . T_{l}^{-1}$ plane when the cognition-level of the single-trial analysis and DT is expressed on the plane. It should be noted that the sampling-frequency is $s_{f}$, so a cycle of $s_{t}(=1000 / f)[\mathrm{ms}]$ is assumed. When $t_{A}=4 i[\mathrm{~ms}]$ and $t_{B}=4 j-x[\mathrm{~ms}]$, then

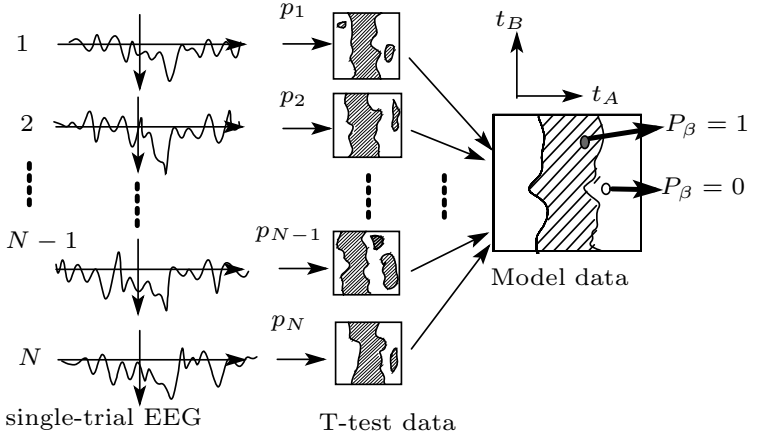

Fig. 3. Determining the model data

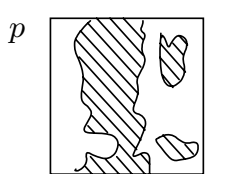

T-test figure

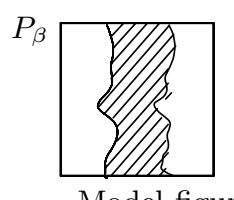

Model figure

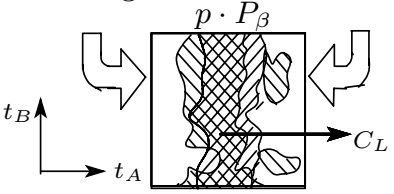

Fig. 4. Determining $C_{L}$

$$
\begin{gathered}
i=0,1, \cdots,(x-\tau) s_{t}, j=0,1, \cdots,(x-\tau) s_{t} \text { in Eq.(3). } \\
P_{\beta}\left(t_{A}, t_{B}\right)=\left\{\begin{array}{cc}
1, & \text { if } \frac{1}{N} \sum_{k=1}^{N} p_{k}\left(t_{A}, t_{B}\right) \geq \beta \\
0, & \text { otherwise } \\
\ldots \ldots \ldots \ldots \ldots \ldots \ldots & (2)
\end{array}\right. \\
C_{L}\left(T_{s}^{-1}, T_{l}^{-1}\right) \\
=\frac{\sum_{i=0}^{(x-\tau) s_{t}} \sum_{j=0}^{(x-\tau) s_{t}} p\left(T_{s}^{-1}, T_{l}^{-1}, i, j\right) \cdot P_{\beta}(i, j)}{\sum_{i=0}^{(x-\tau) s_{t}} \sum_{j=0}^{(x-\tau) s_{t}} P_{\beta}(i, j)}
\end{gathered}
$$

\section{Experiment and Measurement}

The experimental setup is displayed in Fig.5. The modulated vibration is output from the PC's D/Aconverter (Interface Co:PCI-3310), and then the amplitude is amplified to $16 \mathrm{~V}$ by an amplifier. The trigger signal is sent from the D/A-converter to the EEGinstrument (NIHON-CODEN Co:MN-522S) and signals the stimulus starting time. The subject then attaches the PZT (Murata manufacturing Co:77B-21-19RDM$4 \mathrm{AD})$ to his or her forefinger. Each electrode position for the EEG is $\mathrm{Cz}, \mathrm{Fz}, \mathrm{A} 1, \mathrm{~A} 2$. This position refers to the 10-20 system necessary to obtain P300. A1, A2 are the positions required to obtain the base voltage and $\mathrm{Fz}$ is linked to ground. The subject wears earplugs and a headphone that eliminates vibration sounds during the experiment. The subjects should be quiet, seated, maintain closed eyes during the EEG measurements. 
In this paper, $\mathrm{NT}\left(T_{s}^{-1}, T_{l}^{-1}\right)=(120,8)[\mathrm{Hz}]$. However, $\mathrm{TA}$ is an optional modulated vibration on the plane in Fig.6, whose components $T_{s}^{-1}$ and $T_{l}^{-1}$ are set to $40 \leq T_{s}^{-1} \leq 200[\mathrm{~Hz}]$ and $2 \leq T_{l}^{-1} \leq 20[\mathrm{~Hz}]$. The divisions for the $T_{s}^{-1}$ axis is $10[\mathrm{~Hz}]$, while the divisions for the $T_{l}^{-1}$ axis is $1[\mathrm{~Hz}]$.

Figure 7 displays the time schedule for the EEG measurements and the introduction of the stimulus. The stimulus is applied for $2[\mathrm{~s}]$. This report accepts an EEG measured from $-1[\mathrm{~s}]$ to $+1[\mathrm{~s}]$, with $0[\mathrm{~s}]$ representing the time when the stimulus starts. There is no stimulus during Break-time(BT), which is set for $2[\mathrm{~s}]$ in this report. The ratio for supplying the NT or TA stimulus is set to $\mathrm{NT}: \mathrm{TA}=8: 2$, and the order is random. The samplingfrequency $s_{f}$ is $250[\mathrm{~Hz}]$, and the high-cut and low-cut frequencies are $20[\mathrm{~Hz}]$ and $1[\mathrm{~Hz}]$, respectively. The subject is instructed to click the PC-mouse when he or she can recognize the difference between NT and TA. This task is referred to as "RB-task" in this paper.

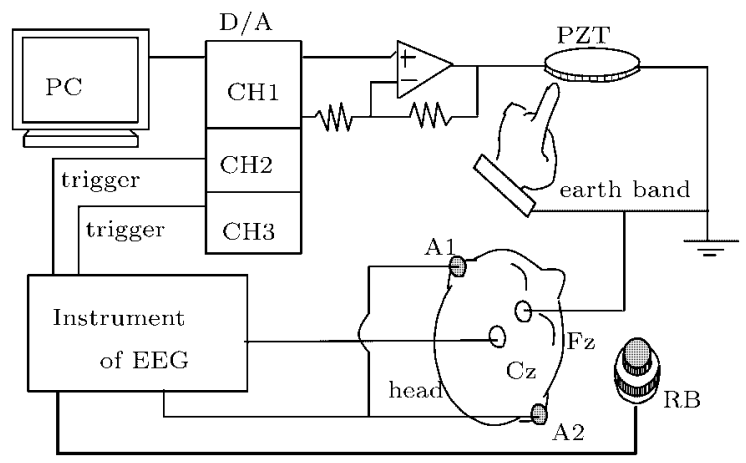

Fig. 5. The experimental setup

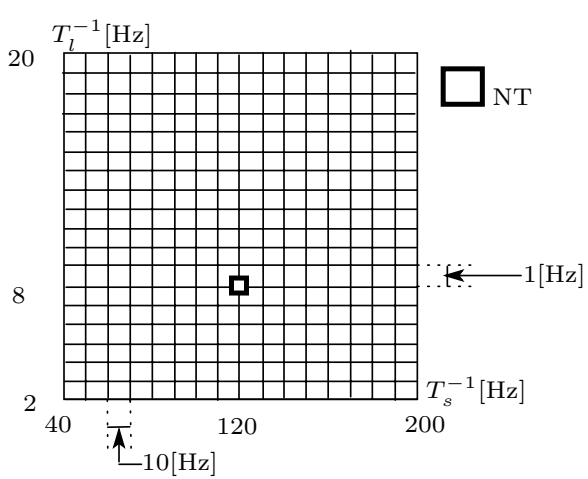

Fig. 6. The frequency-region used

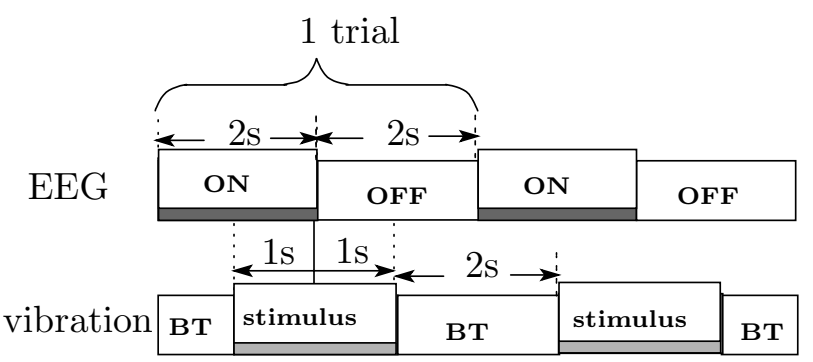

Fig. 7. Measurement time schedule

\section{Results and Discussion}

The experiment was conducted on five subjects(a,b,c,d,e) with a mean age of 22.4 years (S.D. $=2.19$, range $21-26$ ). One subject was female, and the others were male. Parameters $\left(x, \tau, s_{t}, \alpha\right.$, and $\left.\beta\right)$ are set to the following values: $x=1000[\mathrm{~ms}], \tau=200[\mathrm{~ms}], s_{t}=4[\mathrm{~ms}], \alpha=5[\%], \beta=$ 0.8 .

4.1 About the Model-data The model-data $P_{\beta}$ was determined separately for each subject. Fig.8 shows subject(a)'s $P_{\beta}$. In this paper, two types of EEG groups are used to examine the effectiveness of detecting P300 in a single-trial EEG from T-test data. One group has $\mathrm{N}$ single-trial EEGs that can include P300 if the subject was given TA and could answer to RB-task. The other group has $\mathrm{N}$ single-trial EEGs that can include P300 if the stimulus was NT. N was set to 60 . The result for the former are displayed in Fig.8 (a), and the results for the latter are displayed in Fig.8 (b). It should be noted that the $D_{\beta}$ function was assumed using Eq.(4), and its result is presented under the model-data. $D_{\beta}$ is $P_{\beta}$ 's sum when focus is placed on the $t_{B}$ axis at $t_{A}$. When $t_{B}=4 j-x[\mathrm{~ms}]$, then $j=0,1, \cdots,(x-\tau) s_{t}$ in Eq.(4).

Accoding to Fig.8, the belt-shape figure can be found in Fig.8 (a) and a rise of $D_{\beta}$ appears in $300 \leq t_{A} \leq$ $500[\mathrm{~ms}]$. On the other hand, Fig. $8(\mathrm{~b})$ doesn't reflect these features. Fig.9 shows the $D_{\beta}$ values for the other subjects (b,c,d,e). The feature in Fig.8 (a) can be found in Fig.9.

$$
D_{\beta}\left(t_{A}\right)=\sum_{j=0}^{(x-\tau) s_{t}} P_{\beta}\left(t_{A}, j\right)
$$
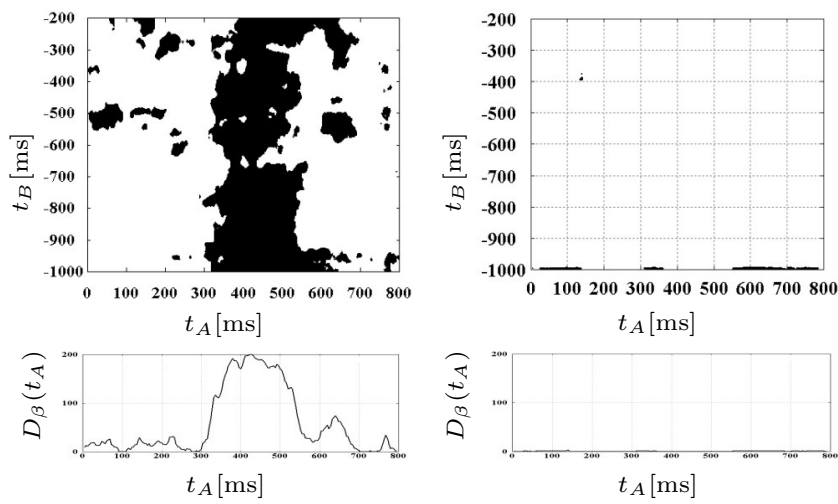

(1) When TA was given

(2) When NT was given

Fig. 8. The model data for subject (a)

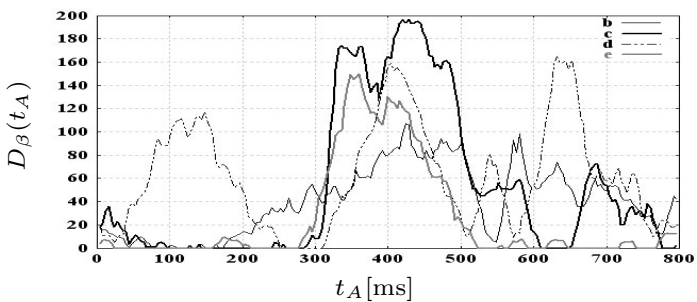

Fig. 9. The results for subjects $(b \sim e)$ show the distinction of P300 in the model data 


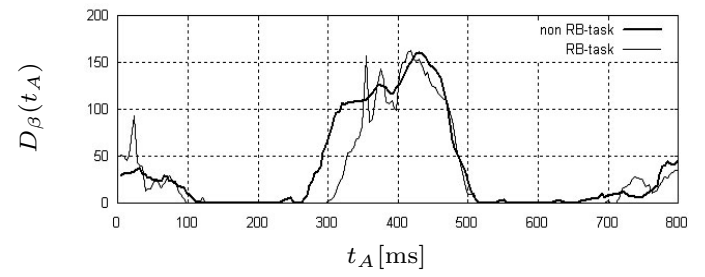

Fig. 10. The influence of MRCP (subject(a))

By the way, it is known that P300 appears in an EEG when a human has cognition of an external stimulus. Previous reports had been recording P300 latency using a tactile-stimulus ${ }^{(12)(13)}$. Thus, two projections can be generated: one feature of Fig.8 (a) or Fig.9 indicates P300 in an EEG, and the other Fig.8 (b) doesn't. In short, the belt shape figure in the T-test data, like that depicted in Fig.8(a), can be an indication of P300 in a single-trial EEG, and the effectiveness of the singletrial analysis can be assured, which was suggested in Section 2.

There is a concern of that hand's MRCP(movementrelated cortical potentials) may have affected EEG. MRCP is the potintials which is related by voluntary movement. Hand's motor area is $7 \mathrm{~cm}$ away from center on the line which links $2 \mathrm{~cm}$ behind of $\mathrm{Cz}$ and external ear canal. LHM and RHM positins are generally used in the hand's MRCP measurment ${ }^{(14)}$.

In this study, results of experiment without RB-task was compared with the one of the experiment with RB-task. This experiment was done about 2 stimulus discrimination easily, $\mathrm{NT}\left(T_{s}^{-1}, T_{l}^{-1}\right)=(120,8) \mathrm{Hz}$, $\mathrm{TA}\left(T_{s}^{-1}, T_{l}^{-1}\right)=(40,8) \mathrm{Hz}$ and $\mathrm{N}=30$. If MRCP affects EEG, the difference will appear in them. The experiment was conducted on subject(a). The example of this experiment ruslut is shown in Fig.10

The result of experiment without RB-task is showed with bold line, and the other one is showed with fine line. According to Fig.10, the striking difference is not assured in their results.

4.2 About the DT Previous reports have reported a peculiarity in DT on the $T_{s}^{-1} v s . T_{l}^{-1}$ plane. The peculiarity is that the cognition level to TA, which is far from NT on the plane, is higher than its level to TA, which is close to NT. A human can easily distinguish between NT and TA because they are widely disparate stimuli. But in the opposite case, such as when TA is close to NT, a human not capable of distinguishing between the two.

In this study, it is assumed that $A_{L}$ function is calculated using Eq.(5) if the subject answers to RB-task $A_{L}=1$, otherwise $A_{L}=0 . \widehat{A}_{L}$ in which $A_{L}$ was smoothed on the $T_{s}^{-1}$ vs. $T_{l}^{-1}$ plane was used as the cognition-level for the RB-task.

$$
A_{L}\left(T_{s}^{-1}, T_{l}^{-1}\right)= \begin{cases}1, & \text { if the subject answered } \\ 0, & \text { otherwise }\end{cases}
$$

Figures 11-13 are results for subjects (a), (b), and (d) for the single-trial analysis (a) and psychological

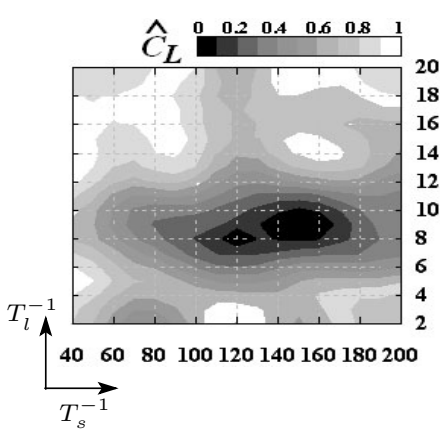

(a) Single-trial analysis

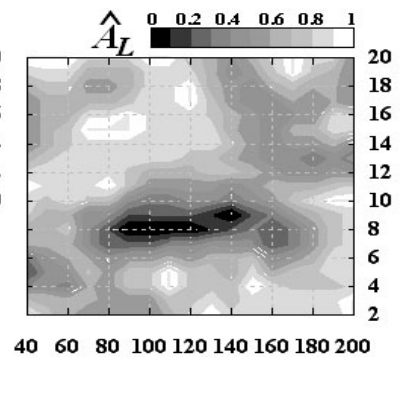

(b) The psychophysical measurement

Fig. 11. The subject (a)'s DT

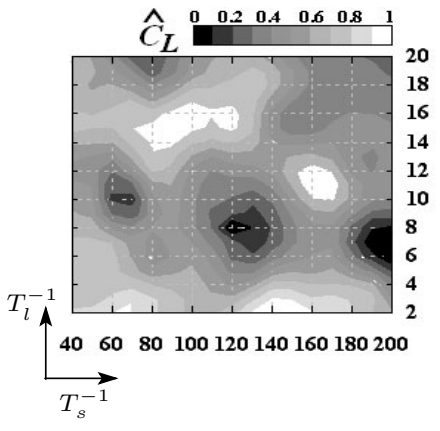

(a) Single-trial analysis

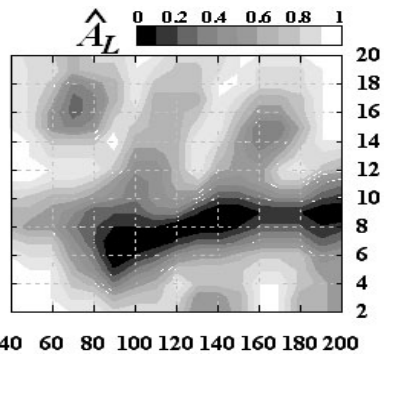

(b) The psychophysical measurement
Fig. 12. The subject (b)'s DT

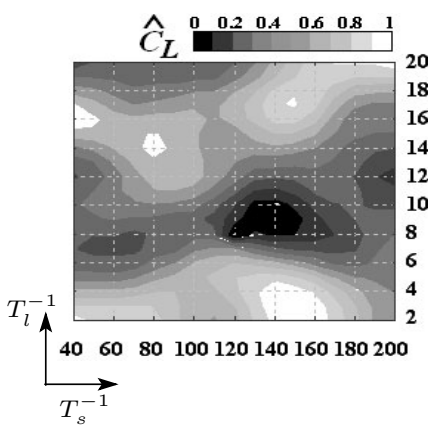

(a) Single-trial analysis

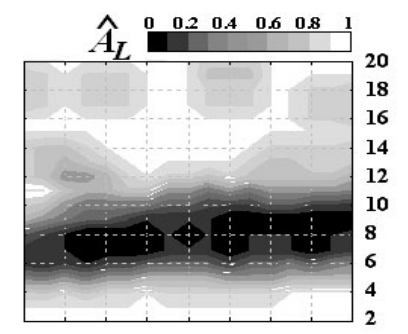

406080100120140160180200

(b) The psychophysical measurement
Fig. 13. The subject (d)'s DT

measurement (b). It is evident from these figures that subject (a)'s $D_{\beta}$ is level is high, subject (b)'s $D_{\beta}$ is level is low, and subject (c)'s $D_{\beta}$ height is a medium level for $300 \leq t_{A} \leq 500[\mathrm{~ms}]$.

Cognition-levels $\widehat{C}_{L}$ and $\widehat{A}_{L}$ are expressed as the black and white colors in the figure. The black color represent a low level, which the white color represents a high level. In other words, the black color indicates that it was difficult for the subject to distinguish between NT and TA, and the white level indicates that the subject could easily distinguish between NT and TA.

The peculiarity cited in previous reports can be observed in Figs.11-13.

4.3 The Effectiveness of the Proposed Analysis The correlation coefficient between $\widehat{C}_{L}$ and $\widehat{A}_{L}$ is 
Table 1. Correlation Coefficient $R$ between $\widehat{C}_{L}$ and $\widehat{A}_{L}$

\begin{tabular}{||c|c||c|c||}
\hline \hline subject & $\mathrm{R}$ & subject & $\mathrm{R}$ \\
\hline \hline $\mathrm{a}$ & 0.47 & $\mathrm{~d}$ & 0.6 \\
\hline $\mathrm{b}$ & 0.34 & $\mathrm{e}$ & 0.48 \\
\hline $\mathrm{c}$ & 0.68 & \multicolumn{2}{|c}{} \\
\hline
\end{tabular}

generated as an $R$ function by using Eqs. (6)-(10). In this calculation, $T_{s}^{-1}=40+10 i[\mathrm{~Hz}], T_{l}^{-1}=2+j[\mathrm{~Hz}]$, $i=0,1, \cdots, n, j=0,1, \cdots, m$. Then, $n=17, m=19$ and $M=n \times m=323$ is set in this section. The result of $R$ is displayed in Table 1 .

$$
\begin{aligned}
& \overline{C_{L}}=\frac{1}{M} \sum_{i=0}^{n} \sum_{j=0}^{m} \widehat{C}_{L}(i, j) \ldots \ldots \ldots \ldots \ldots \ldots \\
& \overline{A_{L}}=\frac{1}{M} \sum_{i=0}^{n} \sum_{j=0}^{m} \widehat{C}_{L}(i, j) \ldots \ldots \ldots \ldots \ldots \\
& X_{\widehat{C}_{L}}(i, j)=\widehat{C}_{L}(i, j)-\overline{C_{L}} \ldots \ldots \ldots \ldots \ldots \\
& Y_{\widehat{A}_{L}}(i, j)=\widehat{A}_{L}(i, j)-\overline{A_{L}} \ldots \ldots \ldots \ldots \\
& R=\frac{\sum_{i=0}^{n} \sum_{j=0}^{m} X_{\widehat{C}_{L}}(i, j) \cdot Y_{\widehat{A}_{L}}(i, j)}{\sqrt{\sum_{i=0}^{n} \sum_{j=0}^{m} X_{\widehat{C}_{L}}^{2}(i, j) \sum_{i=0}^{n} \sum_{j=0}^{m} Y_{\widehat{A}_{L}}^{2}(i, j)}}
\end{aligned}
$$

Generally, coefficient of correlation $R$ can interpret $|R|>0.4$ with correlation of the middle degree. According to Table 1 , four out of the five subjects were determined to have a medium height $R$, that is, $\widehat{C}_{L}$ can correlate with $\widehat{A}_{L}$. Thus the analysis proposed in Section2 can be used to estimate DT.

Now, the discussion is focused on the results of subjects (a) and (b) because their $R$ values are lower than that of the other subjects. Subjects (a) and (b) were given another experiment to obtain their averaged EEG. NT and TA for these trials were the same as those specified in Section 3. However, $T_{s}^{-1}$ or $T_{l}^{-1}$ of TA is set to a constant $120[\mathrm{~Hz}]$ and $8[\mathrm{~Hz}]$, respectively.

The result of the averaged analysis is shown in Fig.14 and Fig.15. The $V_{L}$ function represents the cognitionlevel to the averaged analysis, and the method employed in previous reports was used to calculate $V_{L}$. Fig.14 is the result when $T_{s}^{-1}$ is constant at $120[\mathrm{~Hz}]$, and the result when $T_{l}^{-1}$ is constant at $8[\mathrm{~Hz}]$ is shown in Fig. 14 . In this paper, if the $\widehat{C}_{L}$ and $V_{L}$ values correspond in the figures, then $V_{L}$ is normalized. In the figures, $\widehat{C}_{L}$ is a depicted as a bold black line, and $\widehat{V}_{L}$ is a normal black line. The arrow indicates the position of NT on the $T_{s}^{-1}$ axis.

According to the figures, it is discovered that $\widehat{C}_{L}$ can correlate with $V_{L}$.

Now, $R$ can be calculated between $\widehat{C}_{L}$ and $V_{L}$ by replacing $\widehat{A}_{L}$ with $V_{L}$ in Eqs. $(7),(9),(10)$ for each case. In the former case, $T_{s}^{-1}=120[\mathrm{~Hz}], n=1, m=17, M=17$, and in the latter case $T_{l}^{-1}=8[\mathrm{~Hz}], n=19, m=1, M=19$.

The result is displayed in Table 2 reveals that almost

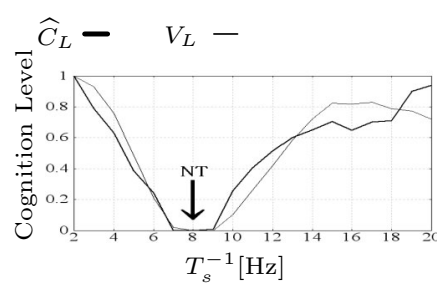

(1) Subject (a)

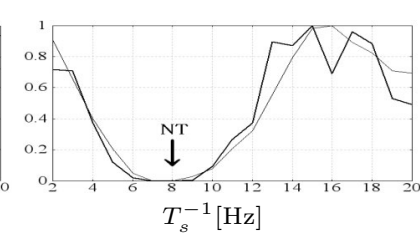

(2) Subject (b)
Fig. 14. Results of $\widehat{C}_{L}, V_{L}$ when $T_{s}^{-1}=120[\mathrm{~Hz}]$

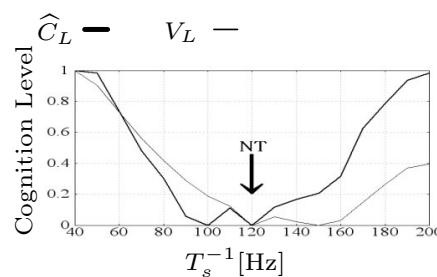

(1) Subject (a)

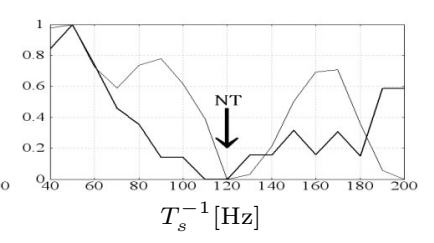

(2) Subject (b)
Fig. 15. Results of $\widehat{C}_{L}, V_{L}$ when $T_{l}^{-1}=8[\mathrm{~Hz}]$

Table 2. Correlation Coefficient $R$ between $\widehat{C}_{L}$ and $V_{L}$

\begin{tabular}{||c|c|c||}
\hline \hline subject & $\left.R\right|_{T_{s}^{-1}=120[\mathrm{~Hz}]}$ & $\left.R\right|_{T_{l}^{-1}=8[\mathrm{~Hz}]}$ \\
\hline \hline $\mathrm{a}$ & 0.93 & 0.70 \\
\hline $\mathrm{b}$ & 0.92 & 0.36 \\
\hline
\end{tabular}

all $R$ values are high-level. However, subject (b)'s $R$ value for the latter case is low-level and $\widehat{C}_{L}$ in Fig.15(b) reflects the peculiarity that has been obtained in previous studies rather than $V_{L}$.

\section{Conclusion}

This paper addresses the DT of frequency for a human's tactile sensor about the modulated vibration reported by previous studies that used the psychological or ERP measurement. In this paper, a method that can determine DT using a single-trial analysis of P300 was proposed. T-test theory was used in the analysis based on the assumption that, on average, the P300's voltage should be higher than that of background activity in a single-trial EEG because P300 has been detected at the top of the plus-voltage in averaged EEGs.

The experiment was conducted with five subjects, and the DTs were individually determined. The subjects were given the task of distinguishing stimuli varied between NT and TA, were NT $\left(T_{s}^{-1}, T_{l}^{-1}\right)=(120,8)[\mathrm{Hz}]$ and an optional TA was included.

In Section 4, a belt-shape figure was evident in all five subject model data. This shape is especially remarkable in $300 \leq t_{A} \leq 500[\mathrm{~ms}]$. In short, the effectiveness of manipulating T-test data to detect P300 in single-trial EEGs was assured. In addition, it was discovered that the DT of the proposed method correlates with those reported by previous methods by calculating the correlation coefficients.

According to results, the effectiveness of presuming DT using a single-trial analysis of P300 was assured. Future studies will focus on detecting DT for various tasks using the method proposed in this report, and the practical use of the tactile-display that employs modulated vibration will be developed. 
Lastly, the authors would like to thank all of the people for their cooperation.

(Manuscript received Aug. 9, 2005, revised Nov. 30, 2005)

\section{References}

(1) M. Uchida, H. Tanaka, H. Ide, and S. Yokoyama: "A Tactile Display by 16 kinds of modulated wave vibration Used Only One PZT Vibrator", T. IEE Japan, Vol.120-C, No.6, pp.825830 (2000-6) (in Japanese)

(2) W. Sommer, J. Matt, and H. Leuthold: "Consciousness of attention and expectancy as reflected in event-related potentials and reaction times", J. Experimental Psychology Learning, Memory \& Cognition, Vol.16, pp.902-915 (1990)

(3) M. Fukuda and M. Uchida: "A Basic Caracteristic for Presentation of 3-D Position Information using PZT Vibrator", T. IEE Japan, Vol.119-C, No.7, pp.915-916 (1999-7) (in Japanese)

(4) G.D. Dawson: "A Summation Technique for the Detectin of Small Evoked potentials", Electroenceph Clin Neutro Physiol, Vol.6, 65/84(1954)

(5) S. Sutton, M. Braren, J. Zubin, and E.R. John: "Evoked potential correlates of stimulus uncertainty", Science, Vol.150, pp.1187-1188(1965)

(6) T. Shiozaki, M. Uchida, H. Tanaka, A. Nozawa, and H. Ide: "The Evaluation of Cognition to the Modulated Vibration Stimulus by ERP", T. IEE Japan, Vol.122-C, No.9, pp.15671572 (2002-9) (in Japanese)

(7) M. Nakamura, Y. Hayashi, and S. Nishida: "Method for Extracting Single Trial P3 from Child ERP by Using the Mathematical Model", Medical Electronics \& Biological Engineering, Vol.33, No.2, pp.135-138 (1995) (in Japanese)

(8) H.S. Liu, T. Zhang, and F.S. Yang: "A Multistage, Multimethod Approch for Automatic Detection and Classification of Epileptiform EEG", IEEE Trans. Biomed. Eng., Vol.49, No.12, pp.1557-1566 (2002)

(9) M. Naoko, M. Uchida, H. Tanaka, A. Nozawa, and H. Ide: "A Study in the difference evaluation of Modulated Vibrarion Using a Single-Trial Waveform of ERP", The Papers of Technical Meeting on Instrumentation and Measurement, IEE Japan, IM-02-82, pp.19-22 (2002-12) (in Japanese)

(10) M. Nakamura, S. Nisida, and H. Sibasaki: "Deterioration of average evoked potential waveform due to asynchronous averaging and its compensation", IEEE Trans. Biomed. Eng., Vol.38, No.3, pp.309-312 (1991)

(11) H. Takagi: Statistics in Clinical Studies- (I) Methods of the Comparison for the Independent Samples, Vol.40(7), pp.559564, Igaku-Shoin Ltd. (1986)

(12) N. Hotta, M. Uchida, H. Hisaya, A. Nozawa, and H. Ide: "The difference threshold of Modulated Vibration Using a Summantion Technique of ERP", The Papers of Technical Meeting on Instrumentation and Measurement, IEE Japan, IM-03-95, pp.67-70 (2003-12) (in Japanese)

(13) A. Magliero, T. Bashore, M.G.H. Coles, and E. Donchin: "On the dependence of P300 latency on stimulus evaluation processes", Psychophysiology, Vol.171, No.6, pp.21-23 (1984)

(14) K. Sato, T. Hirai, and J. Yamaoka: "The Basis and Clinic of Evoked Potentials", pp.209-214, Sozo-Publishing (1990)
Reiko Ohira (Non-member) Graduate Student,Dept. of

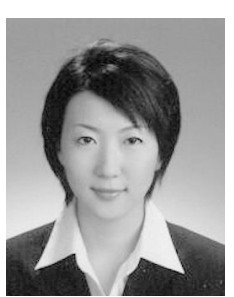
Electronic Eng. The University of ElectroCommunications. (2006 M.A. prospect) Biographical History: 2004 B.A. Dept. of Electronic Eng. The University of ElectroCommunications.

Masafumi Uchida (Member) Associate Professor, Dept. of

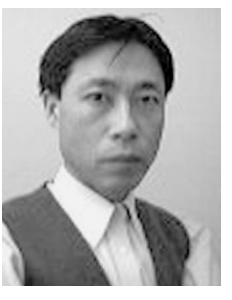
Electronic Eng. The University of ElectroCommunications. Biographical History: 1992 Research Associate, the College of Science and Engineering, Aoyama Gakuin University. 1995 Received a Dr. Eng. degree from Kogakuin University Tokyo, Japan. 1997 Associate Professor, the College of Education, Wakayama University. 2001 Associate Professor, Dep. of Electronic Eng. The University of Electrocommunications. Main Works: Bio-Informatics. and Robotics. Membership in Learned. Societies: RSJ and IEEE.

Akio Nozawa (Member) Research Associate, Dept. of Elec-

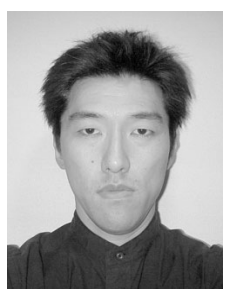
tronics and Electric Eng. Col. of Sci. and Eng., Aoyama Gakuin University. Brief Biographical History: 1997 M.E. Electric and Electronic Engineering, Aoyama Gakuin University. 2002 Research Associate, College of Science and Engineering, Aoyama Gakuin University. Main Works: Bio-Informatics. Membership in Learned Societies: IEICE.

Hideto Ide (Member) Professor, Dept. of Electronics and

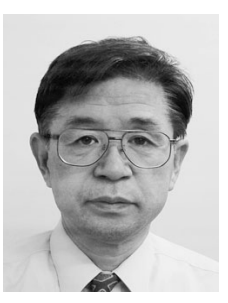
Electric Eng. Col. of Sci. and Eng., Aoyama Gakuin University. Brief Biographical History: 1970 Assistant Professor, College of Science and Engineering, Aoyama Gakuin University. 1985 Professor, College of Science and Engineering, Aoyama Gakuin University. 1996 Dean, College of Science and Engineering, Aoyama Gakuin University. Main Works: Bio-Informatics. Membership in Learned Societies: The Japan Society of Medical Electronics and Biological Engineers and the Society of Instrument and Control Engineers. 